8. Penntube Plastics Product Guide, Clifton Heights, Pa., 19018 (1970).

9. M. C. H. McKubre and D. D. Macdonald, Final Report, U.S. Dept. of Energy Report, Contract EM-78-C-01-5159, Feb. 1980 .

10. B. Miller, This Journal, 116, 1117 (1969).

11. M. C. H. McKubre and D. D. Macdonald, Submitted to This Journal.

12. Y. Beers, "The Theory of Error," Addison-Wesley, Reading, Mass. (1962).

13. W. J. Albery and S. Bruckenstein, Trans. Faraday Soc., 62, 1946 (1966).
14. B. Miller, This Journal, 117, 491 (1970).

15. B. Miller and R. E. Visco, ibid., 115, 251 (1968).

16. H. Schlichting, "Boundary Layer Theory," McGraw Hill, New York (1960).

17. B. Miller, This Journal, 116, 1675 (1969).

18. J. O'M. Bockris and A. K. N. Reddy, "Modern Electrochemistry," Plenum, New York (1970).

19. D. D. Macdonald, Atomic Energy of Canada Limited Report 4139 (1972).

20. D. D. Macdonald and M. C. H. McKubre, To be published.

\title{
Factors Affecting the Electrochemical Responses of Metal Complexes at Pyrolytic Graphite Electrodes Coated with Films of Poly(4-Vinylpyridine)
}

\author{
Noboru Oyama* and Fred C. Anson* \\ Arthur Amos Noyes Laboratory, California Institute of Technology, Pasadena, California 91125
}

\begin{abstract}
Electrochemical responses from the reduction of $\mathrm{Ru}$ III (edta) coordinated to films of high molecular weight poly (4-vinylpyridine) on pyrolytic graphite electrodes were studied as functions of film thickness, temperature, supporting electrolyte composition, and solvent. Responses at filmed electrodes from metal complexes that do not coordinate to the films were also examined. With films thicker than ca. $1000 \AA$, the current responses are limited by the rates of molecular motions within the films. Penetration of counterions, segmental motion of sections of the polymer chains, and juxtapositioning of pairs of attached metal complexes to facilitate intercomplex electron transfer within the film or combinations of the three are suggested as likely current limiting processes.
\end{abstract}

There has been considerable current interest in the preparation and properties of polymer-coated electrode surfaces (1-12). Examples of polymer coatings that have been described include nonelectroactive polymers $(1,6)$, polymers bearing repeating electroactive groups $(2,3,7,8,11)$, and electroinactive polymeric coatings that become electroactive when metal complexes are attached to them $(4,5,7,9)$. Among the questions that await definitive answers for such coatings is how electron transfer is accomplished between the electrode surface and electroactive sites present in the polymeric films.

Kaufman and Engler (8) have recently proposed that electrons are conducted through rather thick films of a polymeric pyrazoline by electron transfer between the electroactive groups within the polymer. They proposed that such transfer required that the sites of electroactivity be mobile enough to achieve appropriate juxtapositions of neighboring sites in order for electron transfer to occur. In addition, the polymer film must be porous enough to allow penetration by ions of the supporting electrolyte to maintain electroneutrality within the film.

In the present report we address this and related issues for films of poly (4-vinylpyridine) (PVP) attached to pyrolytic graphite electrodes. The versatility of this polymeric ligand in anchoring transition metal complexes to electrode surfaces 'was described recently (9) and it seemed desirable to obtain more information on the electron transfer properties of these films which have electroactive groups (metal complexes) introduced into them after they have been attached to the graphite surface.

\section{Experimental}

Pyrolytic graphite electrodes with the graphitic basal planes exposed to the solutions were prepared and treated as previously described (9). Glassy carbon electrodes were prepared by cutting disks from

- Electrochemical Society Active Member.

Key words: polymers, modified electrode, surfaces. cylindrical stock (Tokai Electrode Manufacturing Company, Limited, Tokyo). The disks were sealed to the ends of glass tubing with heat-shrinkable polyolefin tubing (Alfa Wire Company) to produce an electrode area of $0.2 \mathrm{~cm}$. The glassy carbon surface was polished with silicon carbide paper of successively finer grain and finally with $0.3 \mu$ alumina. The polished surface was washed with excess water and methanol.

Poly (4-vinylpyridine) (Borden Incorporated, Philadelphia, Pennsylvania) was recrystallized twice from methanol-diethyl ether. It average molecular weight was determined viscometrically (13) to be $7.4 \times 10^{5}$. This was the polymer utilized in all experiments except those associated with Fig. 2. Aquoethylenediaminetetraacetatoruthenium (III) was prepared and purified as previously described (9). Other chemicals were reagent grade and were used as received. Aqueous solutions were prepared with triply distilled water. The supporting electrolyte for most electrochemical measurements in aqueous media was $0.2 \mathrm{M} \mathrm{CF} \mathrm{COONa}_{3}$ adjusted to the desired $\mathrm{pH}$ with $\mathrm{CF}_{3} \mathrm{COOH}$. Potentials are quoted with respect to a sodium chloride saturated calomel reference electrode (SSCE).

The electrochemical instrumentation and procedures utilized have been described previously (9). With electrodes bearing heavy coatings of PVP and $\mathbf{R u}$ III (edta) the quantity of electrochemically active attached complex could not be determined reliably by electronic integration of voltammograms. Figure 1 shows an example of a voltammogram for a coated electrode and its area as measured by electronic integration of the current during the potential scan. Note that the slope of the integrated current curve does not drop to background levels even at potentials well negative of the peak potential. Significant contributions to the current integral continue to accumulate at potentials far removed from the peak. For this reason the quantities of reactants attached to polymer layers were evaluated by integration of the current that passed when the electrode potential was stepped and held at a value well beyond the voltammetric 

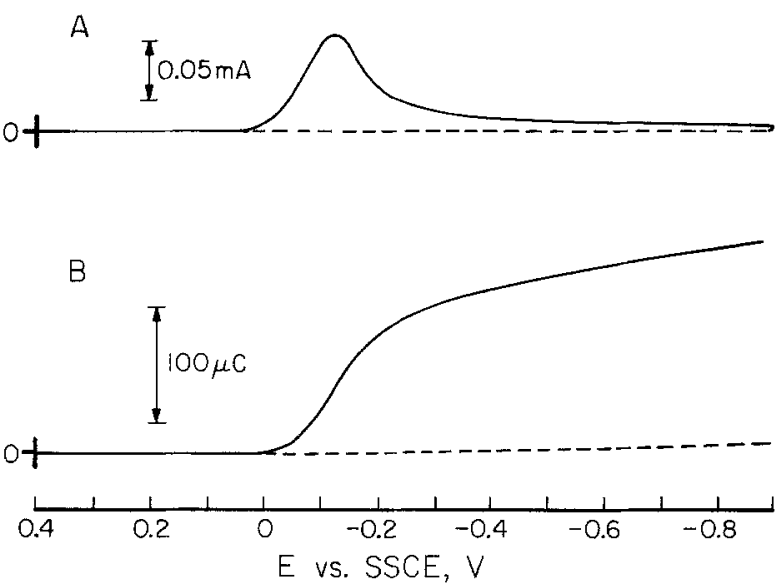

Fig. 1. A, voltammogram for the reduction of RuII (edta) attached to a coating of $2.3 \times 10^{-7}$ moles $\mathrm{cm}^{-2}$ of pyridine os PVP on a pyrolytic graphite electrode. RuIII (edta) attachment wos that resulting when the PVP-coated electrade was exposed to a 5 mmole solution of RuIII (edta) at pH 3.9 for 15 min. Supporting electrolyte: $0.2 \mathrm{M} \mathrm{CF} \mathrm{COONa}_{3}(\mathrm{pH}=2.85)$. Scan rate: $100 \mathrm{mV}$ $\sec ^{-1}$. B, area under the voltammogram in $A$ obtained by electronic integration of the current as the voltammogram was recorded. Background current and its integral are shown by the dashed lines.

peak potential until the rate of charge accumulation decreased to background levels.

To provide electrode surfaces containing known quantities of attached PVP, measured aliquots of a stock solution of the polymer in methanol were carefully transferred to the surface of a horizontally mounted electrode with a microliter syringe. 1-4 microliters of the solution spread uniformly on pyrolytic graphite and well-polished glassy carbon surfaces without overflowing, to produce a visibly uniform film upon evaporation of the solvent. To introduce $\mathrm{Ru} I \mathrm{II}$ (edta) on to the electrode surfaces the resulting polymer-coated electrodes were immersed in a 5 mmole aqueous solution of $\mathrm{Ru}^{\mathrm{III}}$ (edta) at $p \mathrm{H} 3.9$ for $15 \mathrm{~min}$. After attachment of the complex by coordination to the pendant pyridine ligands in the polymer coating (9), the electrodes were washed repeatedly with water, dried, and stored. This procedure is not well calculated to ensure a uniform distribution of coordinated $\mathrm{Ru}$ III (edta) throughout the PVP layer. None of the electrochemical experiments performed with these coatings of PVP-Ru(edta) gave indications of highly anisotropic film compositions but it would not be surprising to find that more of the attached complex resided near the electrolyte/film interface than the electrode/ film interface.

Scanning electron micrographs were obtained with an ETEC Corporation Autoscan U-1 (Hayward, California) instrument. Electrodes were coated by the procedure just described. Cross-sectional views of coatings applied to polished glassy carbon electrodes were obtained by appropriate mounting of the electrode specimens in the microscope. In this way the thickness of the polymeric layers could be estimated.

\section{Results and Discussion}

Effects of molecular weight on the adsorption of PVP on graphite.-To assess the dependence of the molecular weight of the PVP on the extent of its attachment to pyrolytic graphite, a set of PVP samples fractionated by successive crystallization from methanol solutions to which increasing amounts of diethyl ether were added was prepared and the average molecular weight of each fraction was determined viscometrically (13). Freshly cleaved pyrolytic graphite electrodes were exposed to $0.5 \%$ solutions of each fraction in methanol for $15 \mathrm{~min}$ followed by washing with methanol (for $15 \mathrm{~min}$ ) and exposure of the resulting PVP coated electrodes to a 5 mmole solution of $\mathrm{Ru}$ III (edta) for $15 \mathrm{~min}$. The amount of $\mathrm{Ru}^{\mathrm{III}}$ (edta) that coordinated to the PVP coating was then determined electrochemically in $0.2 \mathrm{M} \mathrm{CF}{ }_{3} \mathrm{COONa}(\mathrm{pH} 2.7)$ supporting electrolyte by the methods described in the Experimental section. The extent of RuIII (edta) coordination depended on the average molecular weight of the PVP used to coat the electrode as shown in Fig. 2. The first two points correspond to the use of 4-ethylpyridine and 1,2-bis (4-pyridyl)-ethane to coat the electrode. Neither produced a surface with measurable affinity for $\mathrm{Ru}$ III (edta). With polymers of higher average molecular weight the extent of binding of $\mathrm{Ru}$ III (edta) is assumed to reflect an increase in the quantity of PVP that is present on the graphite surface. PVP samples with average molecular weights in excess of $2 \times 10^{5}$ are clearly the most potent for preparing electrode coatings that accept the largest quantities of RuIII (edta).

Film morphology.-Scanning electron micrographs of films of PVP on glassy carbon show the polymer to be distributed somewhat unevenly with islands of polymer surrounded by bare or lightly covered areas. The uneveness appears to increase when $\mathrm{Ru}$ III (edta) is coordinated to the polymer. A cross-sectional view of the layer produced when a methanolic solution containing $6 \times 10^{-7}$ moles $\mathrm{cm}^{-2}$ of monomeric pyridine units was allowed to evaporate on a glassy carbon surface is shown in Fig. 3. The apparent thickness of the layer is $(8 \pm 2) \times 10^{4} \AA$ which corresponds to an effective density of only ca. $0.08 \mathrm{~g} / \mathrm{cm}^{-3}$. Since the density of bulk poly(4-vinylpyridine) is 1.1 this could indicate that the polypyridine layer is quite porous. However, the apparent film thickness observed in the SEM may not be an accurate reflection of that prevailing on the electrodes used in the electrochemical experiments. For example, several of the experiments to be described in what follows indicate clearly that PVP films deposited on graphite under some conditions behave electrochemically as if they presented a dense, impervious domain to reactants seeking the electrode surface. Thus, exposure of films deposited from methanol to the low pressures within the SEM may cause a large expansion of the film as residual trapped solvent escapes from the film, causing them to appear less dense. For this reason we do not regard the film densities estimated from SEM measurements as representative of electrode surfaces that are never exposed to high vacuum.

Coatings of PVP on glassy carbon that exceed $c a$. $5 \times 10^{-8}$ moles $\mathrm{cm}^{-2}$ (expressed as monomeric pyridine units) are clearly visible without magnification. The originally black surface becomes gray and the glassy carbon loses its luster. When RuII (edta) is coordinated to such heavy PVP coatings, a distinct ard persistent golden color develops on the electrode surface when the $R u$ (III) is reduced to $R u$ (II). The electrode can be cycled repeatedly between potentials that cause reduction and oxidation of the ruthenium

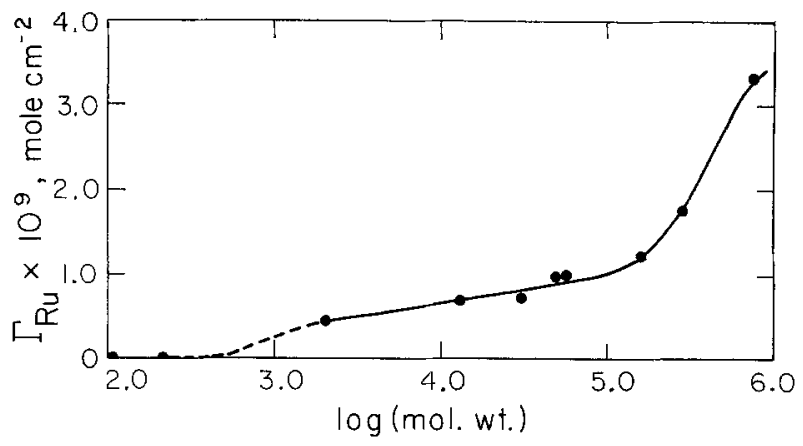

Fig. 2. Effect of the overage molecular weight of PVP on its attachment to grophite electrodes as reflected in the coordination of RuIII (edta) to the polymer coatings under standard conditions (see text for details). 


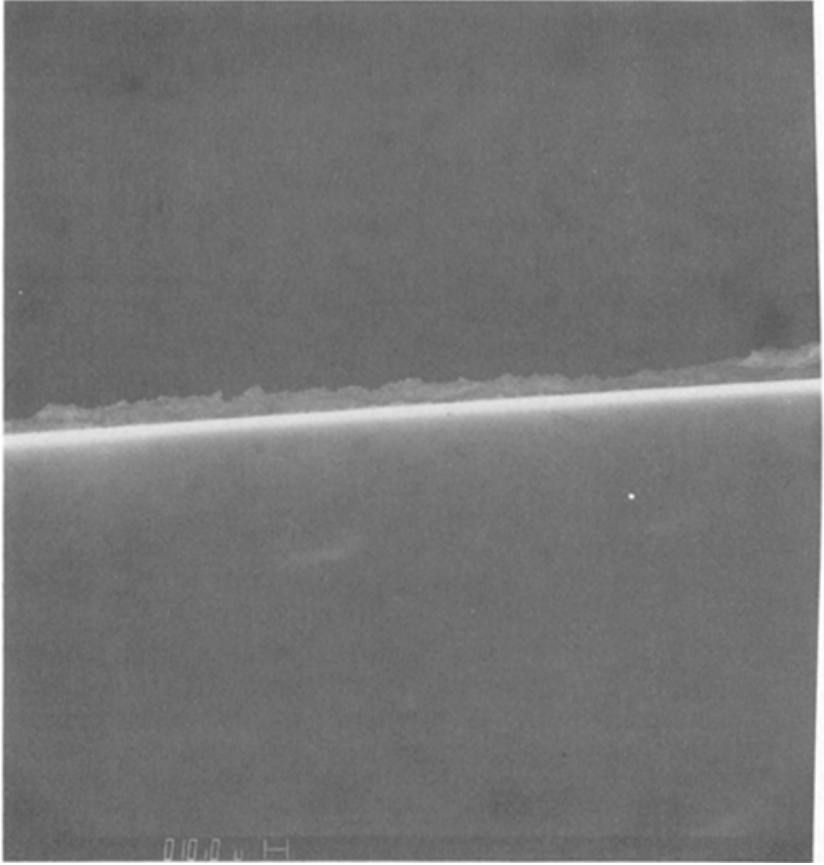

Fig. 3. Scanning electron micrograph of a PVP coating on a glassy carbon electrode. $6 \times 10^{-7}$ moles $\mathrm{cm}^{-2}$ of pyridine as PVP was placed on the surface by evaporation of an aliquot of $a$ methanolic solution. The calibration mark at the bottom of the microgroph corresponds to 10 microns.

centers and the golden color fades and reappears correspondingly. Similar observations have been made with polypyrazoline films (8).

Cyclic voltammetry and chronocoulometry.-Cyclic voltammograms obtained with electrodes coated with PVP to the extent of no more than ca. $10^{-8}$ moles $\mathrm{cm}^{-2}$ (of pyridine units) and then with $\mathrm{Ru}$ (II (edta), by exposure to a $5 \mathrm{mmole}$ solution for $15 \mathrm{~min}$, exhibit the symmetrical shapes and linear dependence of peak currents on scan rate expected for reactants that are attached to electrode surfaces (14). In addition, the area under voltammograms for such coatings shows almost no dependence on scan rate. However, as shown in Fig. 4, increasing the quantity of PVP applied to the electrode surface causes the shapes and peak currents of the resulting voltammograms to change: at first, increasing the quantity of PVP on the electrode surface causes the peak current to increase but still heavier coatings produce diminished peak currents. At the same time the shape of the voltammograms becomes less symmetric and they come to resemble voltammograms for unattached reactants that diffuse to the electrode surface. There is a corresponding change in the scan rate-dependence of the peak currents. With light polymer coatings the peak currents are proportional to the scan rate (Fig. 5) as expected for attached reactants (14) but with sufficiently heavy coatings, the peak currents exhibit the proportionality to the square root of scan rate typical of diffusing reactants (Fig. 5). The apparent areas under the voltammograms obtained with heavy polymer coatings show a strong inverse dependence on scan rate and the integral of the current that passes in response to a potential step from 0.7 to $-0.7 \mathrm{~V}$ grows rapidly at first and then continues to increase slowly for periods as long as 30 or 40 min. Figure 6 compares the charge consumed one minute after a potential step was applied with the charge required to reduce all of the attached complex. With sufficiently heavy coatings of PVP the charge consumed in a fixed time interval is smaller than with lighter coatings even though the actual quantity of RuIII (edta) coordinated to the coating continues to increase as heavier PVP coatings are applied. [This assertion was

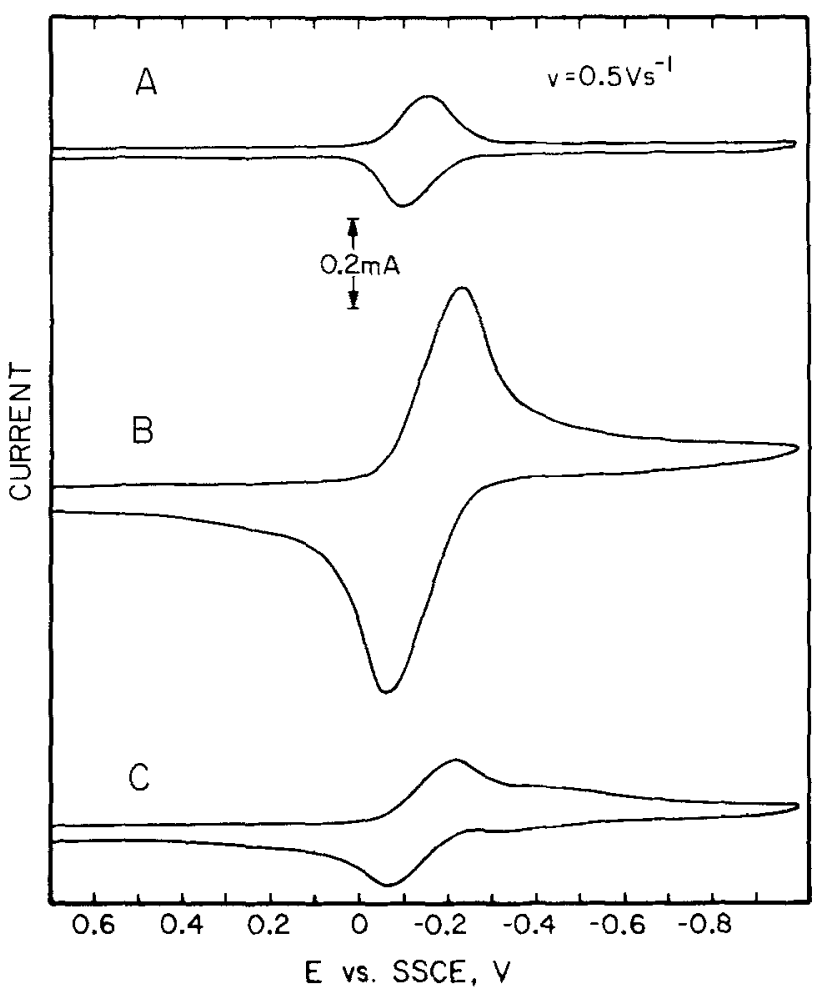

Fig. 4. Cyclic voltammograms for RuIII (edta) attached to pyrolytic graphite electrodes cooted with increasing quontities of PYP. PYP coatings were prepared by evoporation of measured aliquots of solutions of the polymer in methonol. RuIII (edta) attachment procedure os in Fig. 1. Quantity of pyridine introduced on surface os PVP, moles $\mathrm{cm}^{-2}$ : A, $2.3 \times 10^{-8} ; \mathrm{B}, 4.6 \times 10^{-7} ; \mathrm{C}$ $2.3 \times 10^{-6}$. Quantity of $\mathrm{Ru}^{\mathrm{III}}$ (edta) attached, moles $\mathrm{cm}^{-2}: \mathrm{A}$, $2.4 \times 10^{-9} ; B, 1.5 \times 10^{-7} ; C,>3 \times 10^{-7}$. Supporting electrolyte: $0.2 \mathrm{M} \mathrm{CF}_{3} \mathrm{COONo}(\mathrm{pH}=4.2)$. Scan rate: $500 \mathrm{mV} \mathrm{sec}^{-1}$.

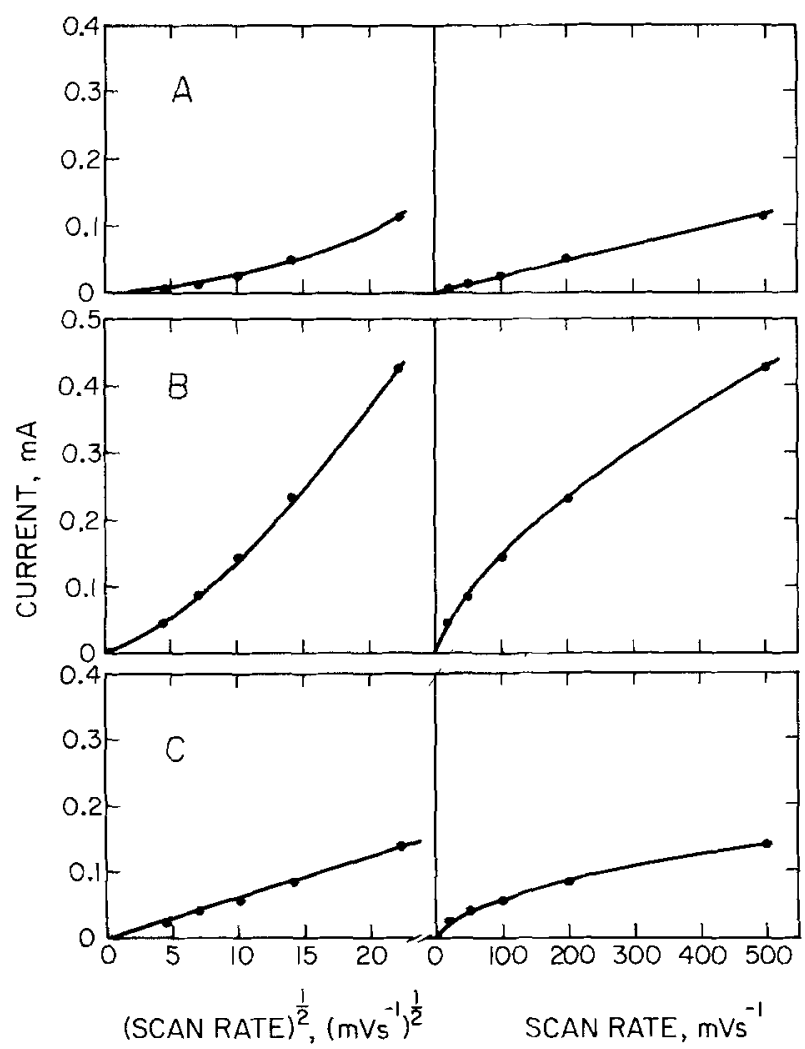

Fig. 5. Peak currents for the three electrodes of Fig. 3 as 0 function of scan rate and (scan rate) $1 / 2$.

confirmed by spectroelectrochemical measurements using optically transparent graphite electrodes $(15,16)$.] 


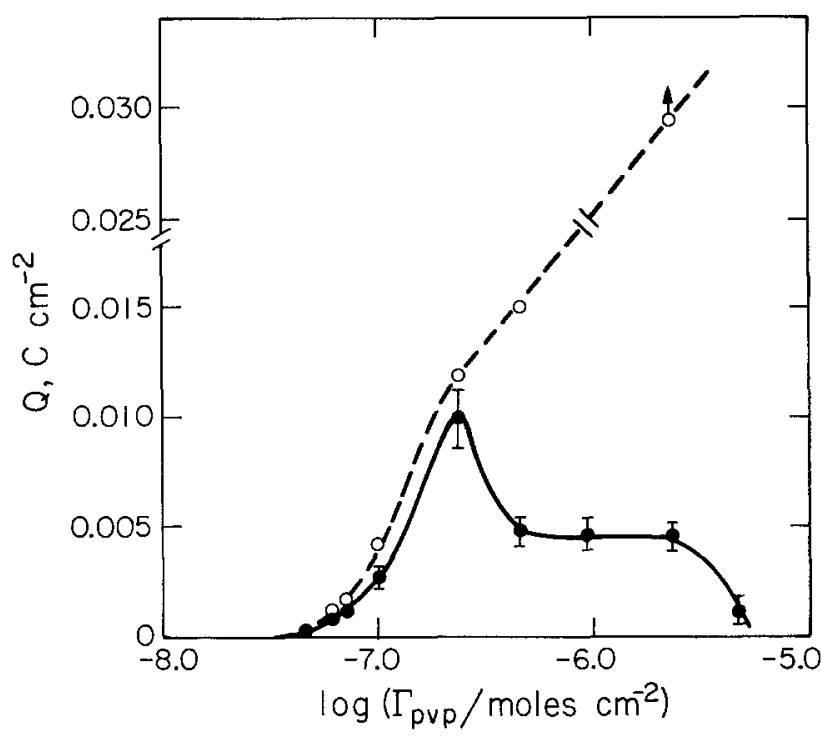

Fig. 6. Charge consumed by the reduction of RuIII (edta) attached to PVP-coated electrodes. (I) Charge consumed during the first $60 \mathrm{sec}$ following the application of a potential step from +0.7 to $-0.7 \mathrm{~V}$. ( $\mathrm{O})$ Charge required for complete reduction of the attached complex at $-0.7 \mathrm{~V}$. Supporting electrolyte: $0.2 \mathrm{M}$ $\mathrm{CF}_{3} \mathrm{COONa}$ ( $\mathrm{pH}=4.2$ ). $\mathrm{Ru}^{\mathrm{III}}$ (edta) attachment procedure as in Fig. 1. The charges required for complete reduction were obtained by continuing the current integration until the rate of increase of of charge matched background levels.

Straight lines were obtained for chronocoulometric plots of the charge passed in response to the potential step against the square root of time. This behavior suggests diffusive transport as the current-limiting factor. In line with the proposals of Kaufman and Engler (8), both the motions of the anchored metal complexes to achieve the proper juxtaposition for intercomplex electron transfer as well as diffusive supply of counterions to the sites where the attached complex is to be reduced could be responsible for the observed behavior. Both the attached $\mathrm{Ru}^{\mathrm{III}}$ (edta) and $\mathrm{Ru}$ II (edta) complexes are likely to be anionic at the $p \mathrm{H}$ of the supporting electrolyte, 4.2 (17). Upon reduction, the negative charge on the complex doubles so that a cation must be supplied to each attachment site before the reduction of the attached $\mathrm{Ru} I I I$ (edta) can occur. The current may be limited by the rate at which segments of the polymer chains within the attached layer can move out of the pathway of counterions entering the film (18). (Note that such motion is required even if the polymer layer contains an abundance of supporting electrolyte because an additional counterion must still be transported from the bulk of the solution to each site where an additional charge is generated.)

Temperature dependence of the electrochemical response.-The diffusion of small molecules (and ions) through polymeric matrices has received considerable study (19-21). Apparent diffusion coefficients that have been measured typically exhibit an Arrhenius type of temperature dependence and the corresponding activation process has been identified with segmental motions within the polymer (20). To evaluate diffusional activation energies for the reduction of the $\mathrm{Ru}^{\mathrm{III}}$ (edta) complex attached to the polymeric layer the temperature dependence of cyclic voltammetric peak currents and chronocoulometric slopes were measured. Figure 7B shows the cyclic voltammograms for $\mathrm{Ru}^{\mathrm{III}}$ (edta) coordinated to a PVP layer on the electrode surface at $5^{\circ}$ and $45^{\circ} \mathrm{C}$. The temperature dependence of the peak currents is comparable to that observed with freely diffusing RuIII (edta). However, since significant quantities of the attached complex can remain unreduced under cyclic voltammetric conditions (Fig. 4), the magnitude of the peak current does not provide a reliable measure of the apparent diffusion coefficient. For this reason we used the slopes of the linear chronocoulometric charge-(time) ${ }^{1 / 2}$ plots shown in Fig. 7A as measures of the product of the (square root of the) apparent diffusion coefficient and the concentration of the diffusing species. It is not straightforward to obtain numerical values of the apparent diffusion coefficients from the slopes of the plots such as those in Fig. 5C and 7A because the concentration of the "diffusing" species is not known. The-concentration of counterions in the bulk of the solution is not the appropriate concentration to use because we observed no change in the magnitude of voltammetric peak currents when the concentration of the supporting electrolyte was varied between 0.02 and $2.0 \mathrm{M}$ at constant $\mathrm{pH}$.

Figure 8 shows the Arrhenius plot of the slopes of the chronocoulometric plots in Fig. 6A from which an apparent diffusional activation energy of $4.6 \mathrm{kcal}$ per
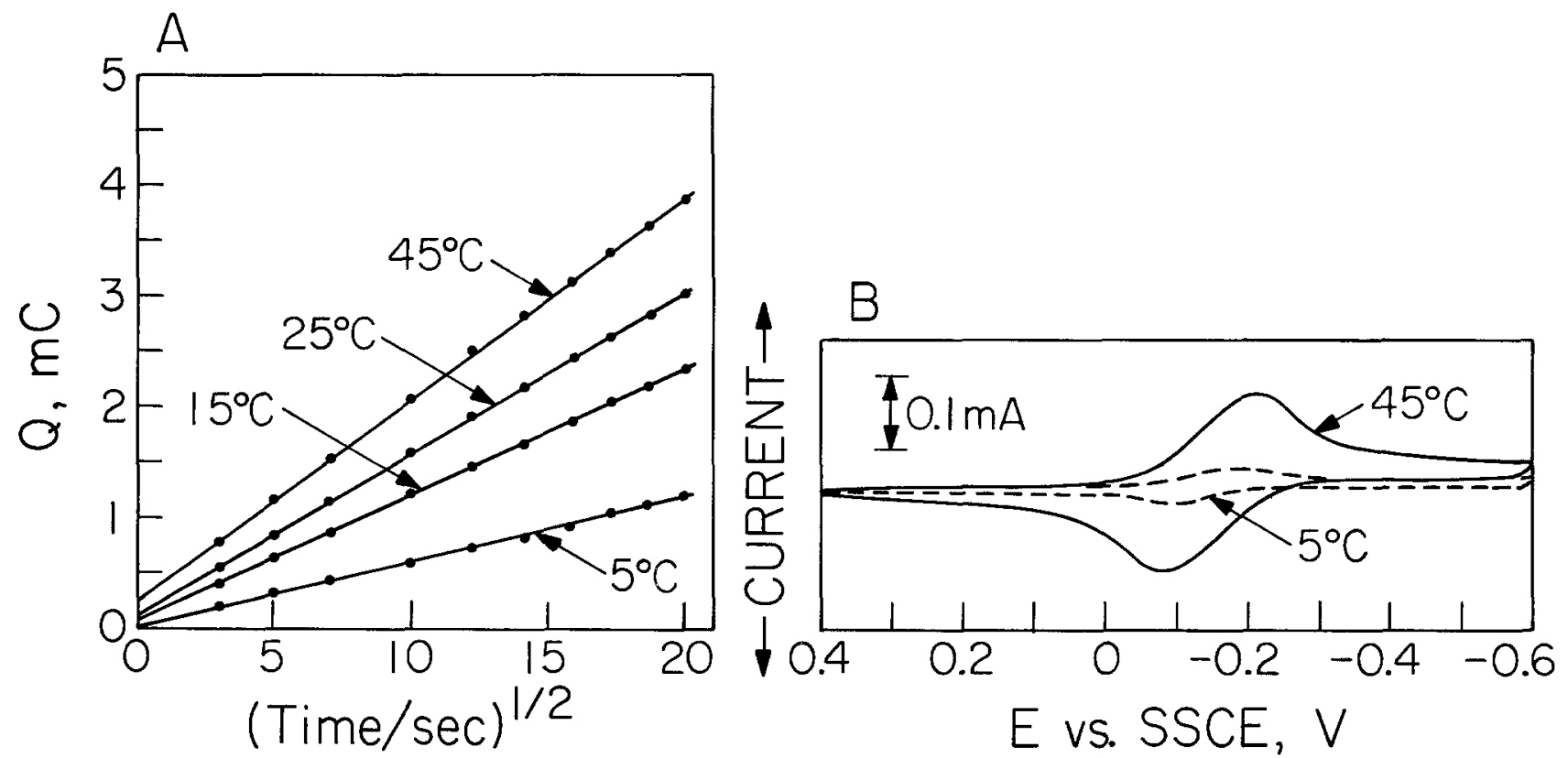

Fig. 7. Temperature dependence of (A), charge-(time)1/2 plots and (B), cyclic voltammograms (scan rate: $200 \mathrm{mV}$ sec -1 ) for $\mathrm{Ru}^{\mathrm{III}}$ (edta) attached to electrodes coated with $2.3 \times 10^{-6}$ moles $\mathrm{cm}^{-2}$ of pyridine as PVP. RuIII (edta) attachment procedure and supporting electrolyte as in Fig. 2 except $\mathrm{pH}$ was 3.4. 


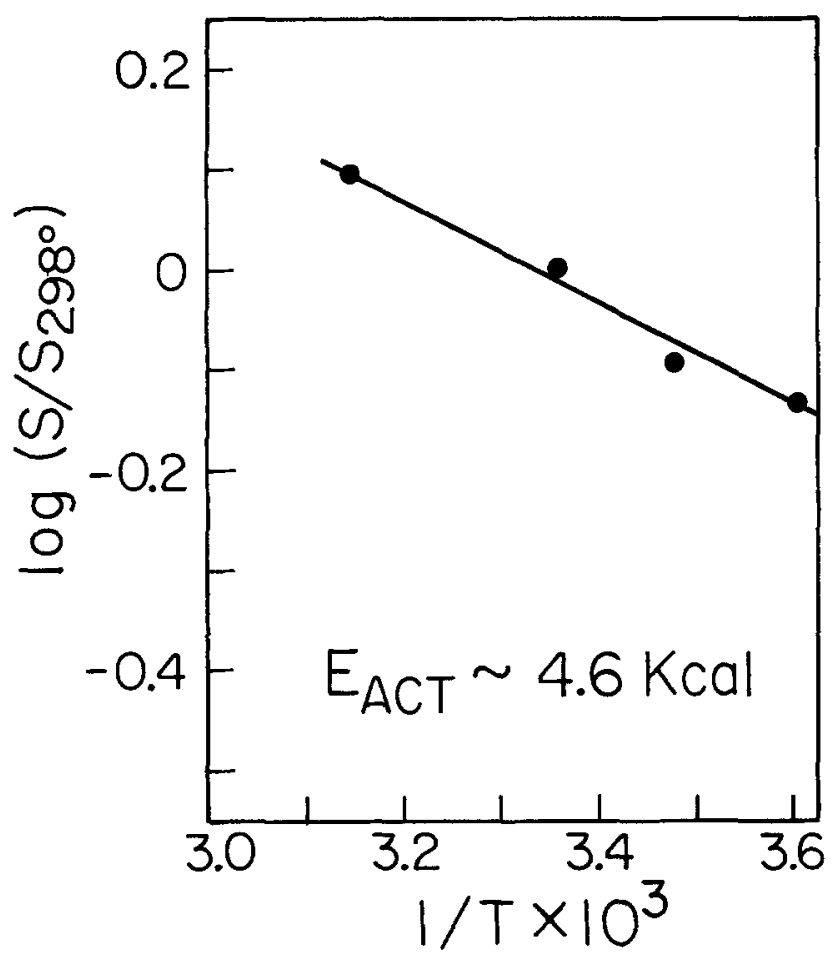

Fig. 8. Arrhenius plot of the slopes of the charge-(time $)^{1 / 2}$ lines in Fig. 7 relative to the slope of $298^{\circ} \mathrm{K}$.

mole was calculated. This is a somewhat smaller value than those reported for the diffusion of small molecules within solid polymer films (20) but it is similar to values observed for such films in the presence of plasticizing agents that facilitate segmental motions of molecular chains within polymeric matrices $(20)$. It does not seem unreasonable that acidic, aqueous solu- tions could serve to "plasticize" the coatings of PVP on graphite surfaces. The activation energy evaluated in Fig. 8 could then be regarded as a measure of the barrier faced by small segments of the PVP chains as they reorient to juxtapose pairs of anchored $\mathrm{Ru}$ (edta) complexes and to permit counterions to reach (or depart form) the sites where the electrode reaction causes an increase (or decrease) in ionic charge.

Effect of solvent on the electrochemical response.Changes in the solvent employed to conduct electrochemical measurements on $\mathrm{Ru} I I I$ (edta) coordinated to PVP coatings can produce dramatic differences in the responses obtained. Figure 8 shows the responses obtained from the same coating in five different solvents. The $\mathrm{Ru}^{\mathrm{III}}$ (edta) is rendered virtually electroinactive in acetonitrile and dimethyl sulfoxide. The effect is not the result of removal of the complex from the surface because transfer of electrodes that give very small responses to an aqueous electrolyte immediately restores the electrochemical activity of the attached complex.

In separate experiments with optically transparent graphite electrodes (15) we have found that essentially all of the $\mathrm{Ru}^{\mathrm{III}}$ (edta) attached to PVP coatings is electroactive in aqueous supporting electrolytes with polymer coatings similar to those employed in Fig. 9. Thus, these results show that changes in solvent can cause a fully electroactive film to become largely inactive.

Solvents that diminish the electroactivity of complexes attached to polymer coatings produce similar effects on electrode process involving unattached reactants: Fig. 10A shows a cyclic voltammogram for ferrocene at an uncoated pyrolyte graphite electrode in acetonitrile. Coating the electrode with a moderately heavy layer of PVP $\left(10^{-7}\right.$ to $10^{-6}$ moles $\mathrm{cm}^{-2}$ of pyridine) somewhat inhibits the ferrocene wave. If

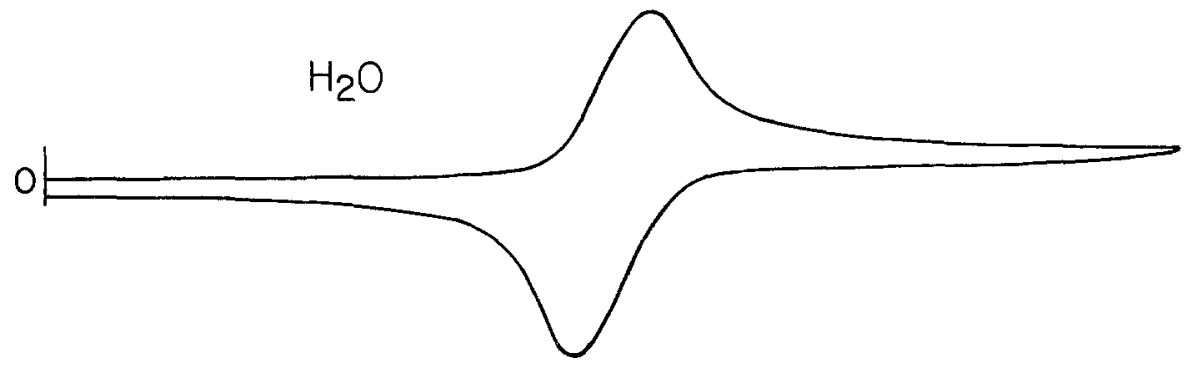

Fig. 9. Solvent dependence of steady-state cyclic voltammograms for RuIII (edta) attached to electrodes couted with $4.6 \times$ $10^{-7}$ moles $\mathrm{cm}^{-2}$ of pyridine as PVP. Supporting electrolytes: $\mathrm{H}_{2} \mathrm{O}, 0.2 \mathrm{M} \mathrm{CF} \mathrm{COONa}_{3}(\mathrm{pH}=$ 4.2); other solvents, $0.2 \mathrm{M}$ $\mathrm{NaClO}_{4}$. Scan rate: $200 \mathrm{mV}$ sec $^{-1}$. RuIII (edta) attachment procedure os in Fig. 2. A freshly cooted electrode was used in each solvent.

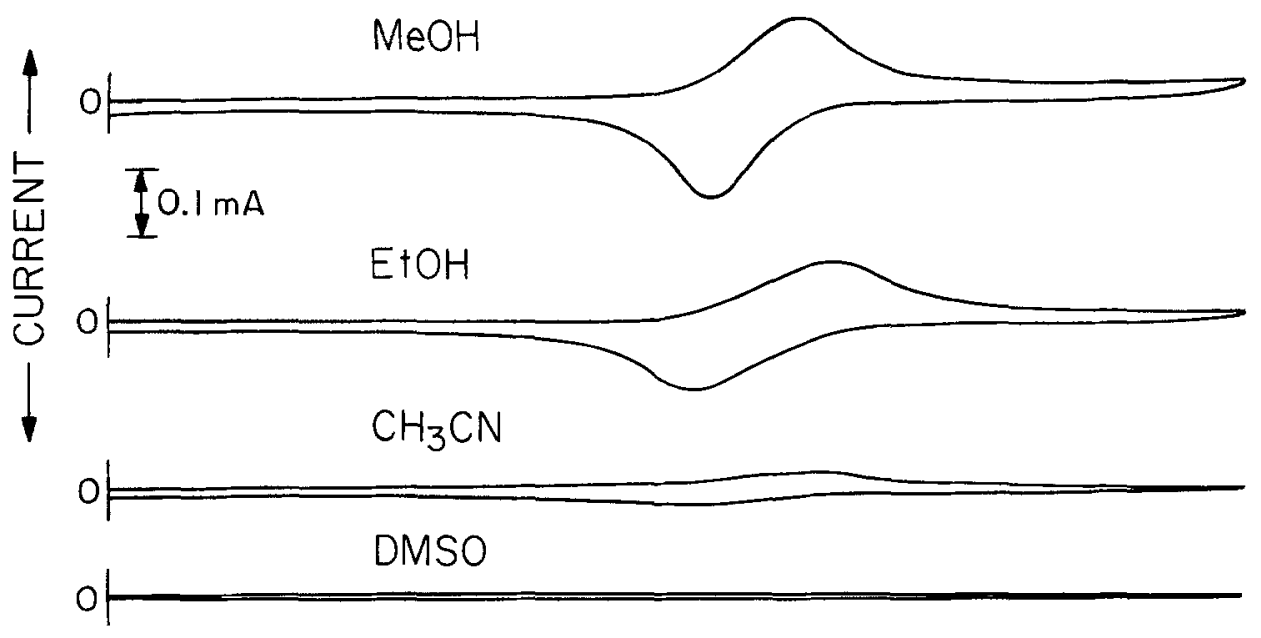

\begin{tabular}{|c|c|c|c|c|c|c|c|c|}
\hline 1 & 1 & 1 & 1 & 1 & 1 & 1 & 1 & 1 \\
\hline 0.6 & 0.4 & 0.2 & 0 & -0.2 & -0.4 & -0.6 & -0.8 & -1 \\
\hline
\end{tabular}



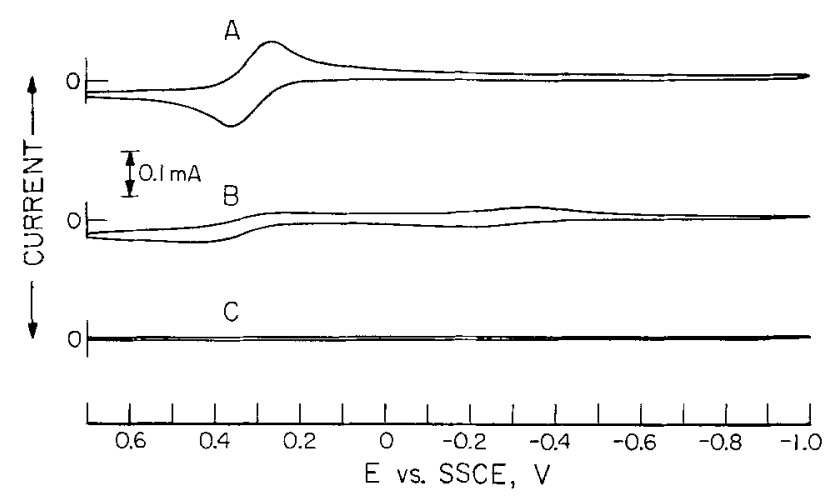

Fig. 10. Steady-state cyclic voltammograms for 1.3 mmole ferrocene at pyrolytic graphite electrodes in acetonitrile. Electrode pretreotments: A, freshly cleaved; $B$, coated with $4.6 \times 10^{-7}$ moles $\mathrm{cm}^{-2}$ of pyridine as PVP, soaked in 5 mmole RuIII (edta) for 15

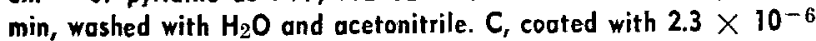
moles $\mathrm{cm}^{-2}$ of pyridine as PVP then as in B. Scan rate: $200 \mathrm{mV}$ sec $^{-1}$. Supporting electrolyte: $0.2 \mathrm{M} \mathrm{NaClO}_{4}$.

RuIII (edta) is attached to the coating, inhibited responses are observed for both the attached complex and ferrocene in the solution (Fig. 10B). With heavier coatings of PVP, the responses from both the attached and the dissolved reactant are severely inhibited (Fig. 10C). That the wave for the dissolved ferrocene can be almost eliminated with sufficiently heavy coatings of PVP indicates that the PVP films produced by exposure to this solvent are relatively free of holes or other defects that would allow the ferrocene to reach the graphite surface. Since the supporting electrolyte would presumably also be unable to penetrate the film, it is not surprising that reactants attached to such films should also exhibit little or no electroactivity. Structures of polymeric films that are compact enough to impede the motion of supporting electrolytes through them might also lack adequate flexibility for the needed positioning of the redox centers to accomplish electron transfer through the film.

The swelling of polymeric matrices bearing ionic groups, e.g., ion exchange resins, by exposure to aqueous media (22) provides a familiar example of the ability of solvents to influence the density and texture of polymeric materials (20). It seems likely that the differences among the voltammograms in Fig. 9 can be attributed in large measure to variations in the swelling tendencies among the solvents tested.

Effects of changes in supporting electrolyte.-Change in the nature of the aqueous supporting electrolytes in which the electrochemistry of attached RuII (edta) is observed produce quantitative differences in the responses obtained rather than the qualitative differences arising from changes in the solvent. Figure 11

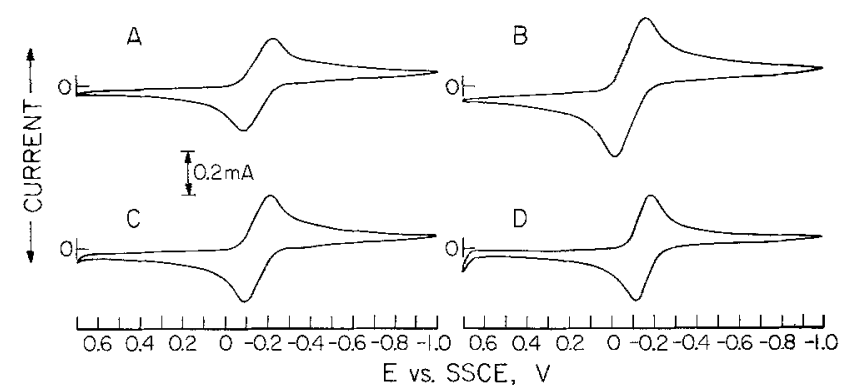

Fig. 11. Effects of supporting electrolyte on steady-state cyclic voltammograms for RuIII (edta) attached to electrodes coated with $2.3 \times 10^{-6}$ moles $\mathrm{cm}^{-2}$ of pyridine as PVP. A, $0.2 \mathrm{M}$ $\mathrm{CF}_{3} \mathrm{COONa}$ ( $\mathrm{pH}=4.2$ ). B, $0.2 \mathrm{M} \mathrm{CF} \mathrm{CFOONa}_{3}$ plus $0.025 \mathrm{M}$ $\mathrm{CF}_{3} \mathrm{COOH}(\mathrm{pH}=1.6)$. C $0.2 \mathrm{M} \mathrm{NaBr}(p \mathrm{H}=4.2)$. D, $0.2 \mathrm{M}$ $\left(\mathrm{CH}_{3}\right)_{4} \mathrm{NBr}(\mathrm{pH}=4.2)$. Scan rate: $200 \mathrm{mV} \mathrm{sec}-1$. Ru' $\mathrm{mII}^{\mathrm{III}}$ (edta) attachment procedure as in Fig. 2. compares cyclic voltammograms recorded in four different supporting electrolytes with identically prepared electrode surfaces. The peak current in the electrolyte at $p \mathrm{H} 1.6$ (curve $\mathrm{B}$ ) is about twice as large as those in the other electrolytes indicating that charge transfer through the film is facilitated by protonation of the pyridine groups. Protonated PVP chains no doubt tend to stretch apart to minimize coulombic repulsions (23) and this would lead to a film through which ions could move more readily. As a result, segmental motions within the polymer layer may also be facilitated because the penetration of macromolecular solids by low molecular weight species is known to produce such effects (24).

Curves $\mathrm{A}$ and $\mathrm{C}$ in Fig. 11 have rather similar shapes indicating that changing the anion of the supporting electrolyte from trifluoroacetate to bromide produces only small effects. The change of supporting electrolyte cation from sodium to tetramethylammonium (curves $\mathrm{C}$ and $\mathrm{D}$ ) yields a more symmetrical voltammogram. This might be the result of less hydration of the latter cation with a corresponding decrease in the extent of segmental motion within the polymer required for its penetration.

The suggestion that the current enhancement in curve B of Fig. 11 results from an "opening up" of the polymeric structure because of repulsive interactions among protonated sites along the polymer chains receives adaed support from the voltammograms in Fig. 12. Curve A in Fig. 12 is a cyclic voltammogram for $\mathrm{Fe}^{\mathrm{III}}$ (edta) at an uncoated electrode. Curve B results when the electrode is coated with PVP and used in the solution of $\mathrm{Fe}^{\mathrm{III}}$ (edta) at a $\mathrm{pH}$ where the PVP is not significantly protonated (25). The polymer layer apparently cannot be penetrated by the $\mathrm{Fe}$ III (edta) complex. However, when the $p H$ is lowered to a value where the PVP layer is protonated, the electroreduction of Fe III (edta) is immediately restored (curve C). When $\mathrm{Ru}\left(\mathrm{NH}_{3}\right)_{6}{ }^{3+}$ is substituted for the anionic $\mathrm{Fe}^{\mathrm{III}}$ (edta) complex and the experiments repeated, curves D, E, and F in Fig. 12 are obtained. Protonation of the PVP film restores only partially the electrochemical response from the reduction of $\mathrm{Ru}\left(\mathrm{NH}_{3}\right)_{6}{ }^{3+}$ (curve F). Protonation of the polymer produces a more porous film but it is also highly positively charged so that the tri-positive reactant evidently can traverse it only with difficulty. This would account for the drawn-out shape of voltammogram $F$ compared with that for the anionic $\mathrm{Fe}^{\mathrm{III}}$ (edta) complex in voltammogram $\mathrm{C}$.

An additional factor that could influence the ionic conductivity of PVP layers is the increase in the quantity of counterions within the polymeric matrix that must accompany the introduction of charged sites. Thus, the quantity of anions that are electrostatically held within a protonated PVP layer will be relatively insensitive to the ionic strength of the supporting electrolyte but highly sensitive to its $p H$. The magnitudes of voltammetric peak currents and chronocoulometric slopes for anchored reactants exhibit similar sensitivities which might be an indication that the counterionic content of the polymer coatings controis the magnitude of the currents that can traverse them.

One is left with two possible rationalizations for the increased currents that result when charged sites are introduced into PVP films: increased permeability arising from stretching of the polymer chains by repulsive electrostatic interactions among sites and/or increased conductivity within the polymeric matrix resulting from a higher salt content. The data presently available do not allow a clear choice to be made between these two rationalizations or rule out the possibility that both mechanisms are operative.

If protonation of PVP films produces a more open texture the introduction of other charged ions into the polymer might be expected to produce similar effects. Figure 13 demonstrates that the attachment of $\mathrm{Ru}$ III (edta) to a PVP film does indeed convert a 
Fig. 12. Steady-state cyclic voltammograms for 5 mmole solutions of $\mathrm{Fe}^{\mathrm{III}}$ (edta) or $\mathrm{Ru}\left(\mathrm{NH}_{3}\right)_{6}{ }^{3+}$ at pyrolytic graphite electrodes under following conditions: A, D, freshly cleaved electrode; supporting electrolyte, $0.2 \mathrm{M} \mathrm{CF}{ }_{3} \mathrm{COONa}$ of $\mathrm{pH} 5.4$ ( $\mathrm{Fe}$ ) or $6.0(\mathrm{Ru})$. B, E, coated with $4.6 \times 10^{-7}$ moles of pyridine os PVP; supporting electrolyte as in $A, D$. C, F, supporting electrolyte adjusted to $\mathrm{pH} 2.2$ with $\mathrm{CF}_{3} \mathrm{COOH}$. The doshed line in curve $C$ is the response of a freshly cleaved electrode al pH 2.2. The response for $\mathrm{Ru}\left(\mathrm{NH}_{3}\right)_{6}{ }^{3+}$ showed no significant $\mathrm{pH}$ dependence. Scan rate: $200 \mathrm{mV} \sec ^{-1}$.

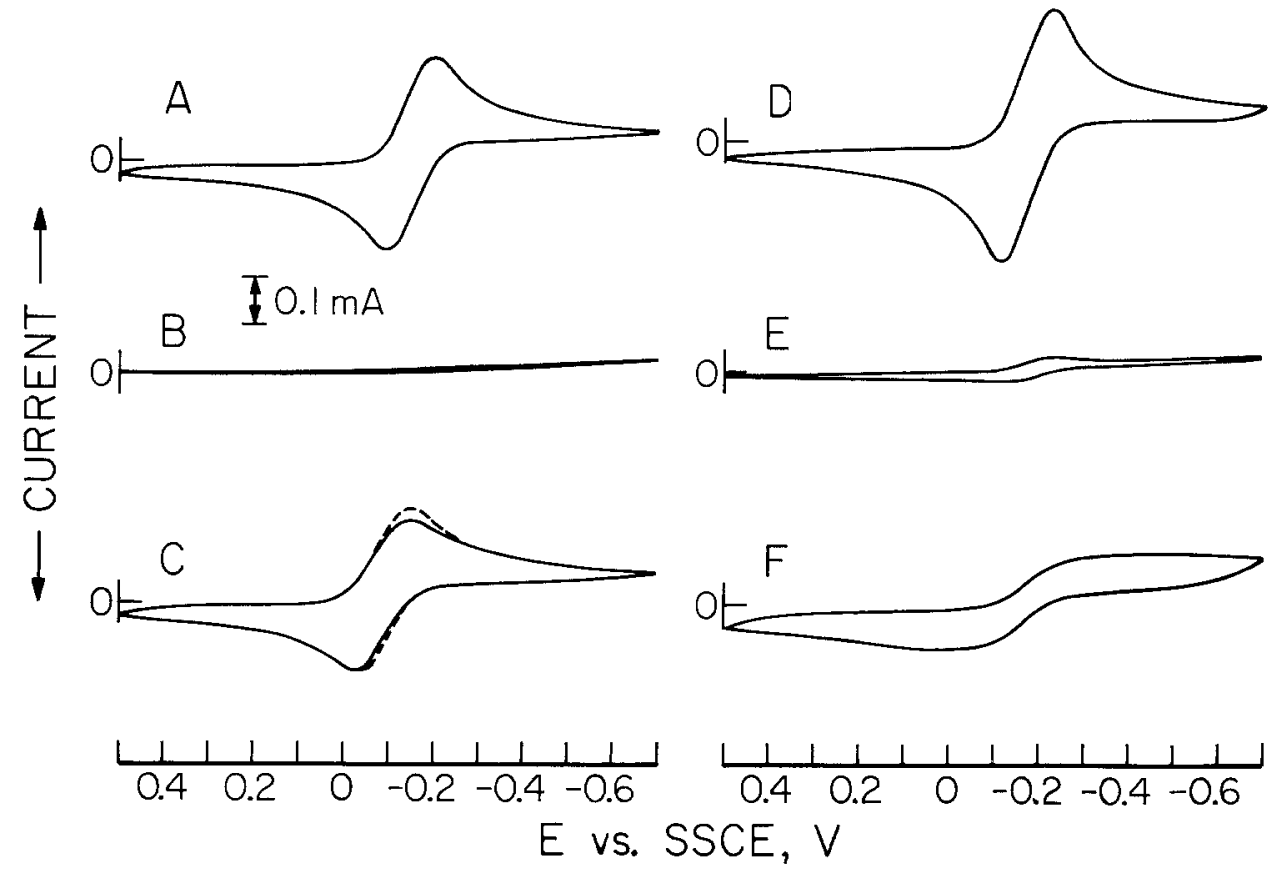

blocking layer into one at which the $\mathrm{Fe}(\mathrm{CN})_{6}{ }^{3-14-}$ redox reaction can proceed. The cyclic voltammogram obtained at an uncoated electrode (curve A) is annihilated by the presence of an unprotonated PVP coating (curve B) but it reappears, in a slightly distorted form, when Rulll (edta) [a monoanion at $\mathrm{pH}$ $5.4(17)]$ is coordinated to the polymer (curve $C$ ). In this case the density of bound (anionic) charge is not as high as that resulting from protonation of the polymer at low $p H$ but the restored response is almost as great as that obtained at the uncoated electrode. The coated electrode can also be unblocked by protonation, as in Fig. 12.

The response observed for the $\mathrm{Fe}(\mathrm{CN})_{6}{ }^{3-14}-$ couple in curve $C$ of Fig. 13 can be understood simply in terms of an increase in the porosity of the film which allows the reactant to pass into the film much more readily than was true under the conditions of curve B. However, a second possible origin of the increased film conductance in this case (as contrasted with curves $\mathrm{C}$ and $F$ in Fig. 12) is the transmission of electrons through the film via the attached $\mathrm{Ru}$ III (edta) centers. That is, electron transfer from the graphite to the $\mathrm{Fe}(\mathrm{CN})_{6^{3-/ 4-}}$ couple may be mediated by electron transfer among the anchored redox centers in the film. Distinguishing between these two mechanisms of

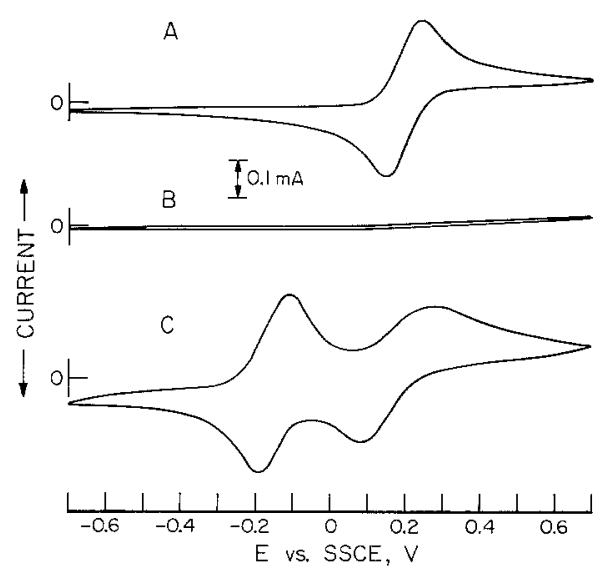

Fig. 13. Steady-state cyclic voltammograms for a $5 \mathrm{mmole}$ solution of $\mathrm{Fe}(\mathrm{CN})_{6}{ }^{4-}$ in $0.2 \mathrm{M} \mathrm{CF}{ }_{3} \mathrm{COONa}$ at $\mathrm{pH}$ 6.0. Pyrolytic graphite electrode pretreatments: $A$, freshly cleaved; $B$, coated with $4.6 \times 10^{-7}$ moles $\mathrm{cm}^{-2}$ of pyridine as PVP; $C$, after the electrode used to record curve $B$ was soaked for $15 \mathrm{~min}$ in 5 mmole RuIII (edta) and rinsed. Scan rate: $200 \mathrm{mV} \mathrm{sec}^{-1}$. charge transfer may be possible by suitable manipulation of mass transfer and charge transfer rates and we have such experiments underway. However, it may be worth emphasizing two points in conclusion. (i) The presence of electroactive redox centers within polymer films is no assurance that the films will support charge transfer either to the attached redox centers or to reactants dissolved in solution: PVP coatings containing large quantities of coordinated RuIII (edta) nevertheless behave as insulating layers in certain nonaqueous solvents (Fig. 10). (ii) Charge transfer between dissolved reactants and electrode surfaces covered with thick $(1-10 \mu \mathrm{m})$ PVP films can occur rapidly even when the films do not have redox centers attached to them: protonation converts an insulating PVP film to one that does not impede the reduction of dissolved reactants (Fig. 12).

\section{Acknowledgments}

The assistance of Prof. J. P. Revel and Pat Cohen in obtaining electron micrographs is acknowledged gratefully. Drs. Alan Rembaum and Roger Williams kindly provided the samples of fractionated PVP that were used to obtain the data for Fig. 2. Several perceptive comments and criticisms by the reviewers of an earlier version of this manuscript helped us to formulate our speculations more clearly.

This work was supported by the National Science Foundation and the U.S. Army Research Office.

Manuscript submitted July 23, 1979; revised manuscript received Oct. 15, 1979.

Any discussion of this paper will appear in a Discussion Section to be published in the December 1980 Journal. All discussions for the December 1980 Discussion Section should be submitted by Aug. 1, 1980 .

Publication costs of this article were assisted by the California Institute of Technology.

\section{REFERENCES}

1. L. L. Miller and M. R. Van de Mark, J. Am. Chem. Soc., 100, 639 (1978); J. Electroanal. Chem. Interfacial Electrochem., 88, 437 (1978).

2. M. R. Van de Mark and L. L. Miller, J. Am. Chem. Soc., 100, 3225 (1978)

3. A. Merz and A. J. Bard, ibid., 100, 3222 (1978).

4. K. Itaya and A. J. Bard, J. Anal. Chem., 51, 1487 (1978).

5. N. Oyama and F. C. Anson, J. Am. Chem. Soc., 101, 739 (1979).

6. K. Dobelhofer, D. Noete, and J. Ulstrup, Ber. Busenges. Phys. Chem., 82, 403 (1978). 
7. R. Nowak, F. A. Schultz, M. Umana, H. Abruna, and R. W. Murray, J. Electroanal. Chem., Interfacial Electrochem., 94, 219 (1978).

8. F. B. Kaufman and E. M. Engler, J. Am. Chem. Soc., 101, 547 (1979).

9. N. Oyama and F. C. Anson, ibid., 101, 3450 (1979).

10. F. B. Kaufman, A. H. Schroeder, E. M. Engler, S. R. Kramer, and J. Q. Chambers, ibid., In press.

11. F. B. Kaufman, A. H. Schroeder, E. M. Engler, J. Q. Chambers, and S. R. Kramer, Abstract 301, p. 778, The Electrochemical Society Extended Abstracts, Boston, Massachusetts, May 6-11, 1979.

12. P. Peerce and A. J. Bard, Abstract 302, p. 781, The Electrochemical Society Extended Abstracts, Boston, Massachusetts, May 6-11, 1979.

13. S. R. Rafikov, S. A. Pavlova, and I. I. Tverdokhlebova, "Determination of Molecular Weights and Polydispersity of High Polymers," Ch. IX, Daniel Davey and Co., Inc., New York (1964).

14. E. Laviron, J. Electroanal. Chem. Interfacial Electrochem., 39, 1 (1972)

15. N. Scott, N. Oyama, and F. C. Anson, J. Electroanal. Chem. Interfacial Electrochem., In press.

16. T. P. DeAngelis, R. W. Hurst, A. M. Yacynck, H. B. Mark, Jr., W. R. Heineman, and J. S. Mattson,
Anal Chem., 49, 1395 (1977).

17. K. Shimizu, T. Matsubara, and G. P. Sato, Bull. Chem. Soc. Jpn., 47, 1651 (1974).

18. J. Frank and G. S. Park, Editors, "Diffusion in Polymers," Chap. 10, Academic Press, New York (1968).

19. J. Frank and G. S. Park, Editors, "Diffusion in Polymers," Chap. 2-10, Academic Press, New York (1968).

20. L. Rebenfeld, P. J. Makarewicz, H. Weigmann, and G. L. Wilkes, J. Macromol. Sci. Rev. Macromol. Chem., Chap. 15, p. 279 (1976).

21. R. McGregor, "Diffusion and Sorption in Fibers and Films," Chap. 15, Academic Press, New York (1974).

22. W. Rieman and H. F. Walton, "Ion Exchange in Analytical Chemistry," Pergamon, New York (1970).

23. H. Morawetz, "Macromolecules in Solution," 2nd ed., Chap. VII, John Wiley and Sons, Inc., New York (1975).

24. R. Kosfeld and L. Zumkley, Ber. Bunsenges, Phys. Chem., 83, 392 (1979).

25. H. Nishikawa and E. Tsuchida, J. Phys. Chem., 79, $2072(1975)$.

\title{
A Digital Simulation Model for Electrochromic Processes at WO Electrodes
}

\author{
Benjamin Reichman and Allen J. Bard* \\ Department of Chemistry, The University of Texas at Austin, Austin, Texas 78712
}

and Daniel Laser

Department of Chemistry, Tel Aviv University, Ramat-Aviv, Israel

\begin{abstract}
Current-potential ( $i-E$ ) curves for the electrochromic process at $\mathrm{WO}_{3}$ electrodes were calculated with a digital simulation model which assigns the rate of charge transfer at the oxide/solution interface and the rate of diffusion of hydrogen into the bulk of the film as major variables. The simulated $i-E$ curves agreed well with experimental ones for different types of $\mathrm{WO}_{3}$ films and predicted the observed dependency of current on scan rate. The simulation required knowledge of the form of the electrochemical isotherm, which was obtained experimentally, and adjustment of a charge transfer rate constant, $k_{f}$, and the hydrogen atom diffusion coefficient within the film, $D_{\mathrm{H}}$. The best fit was obtained with $k_{\mathrm{f}}=9 \times 10^{-3} \mathrm{sec}^{-1}\left(\mathrm{~mole} / \mathrm{cm}^{3}\right)-2$ and $D_{\mathrm{H}}=1 \times 10^{-9}$ to $2 \times 10^{-10} \mathrm{~cm}^{2} / \mathrm{sec}$ for the $\mathrm{WO}_{3}$ films prepared by vacuum evaporation and $k_{\mathrm{f}}=7.2 \mathrm{sec}^{-1}\left(\operatorname{mole} / \mathrm{cm}^{3}\right)^{-2}$ and $D_{\mathrm{H}}=5 \times 10^{-8} \mathrm{~cm}^{2} / \mathrm{sec}$ for $\mathrm{WO}_{3}$ anodic films. Simulated potential step results, which are similar to the experimental curves at longer times but show some discrepancy in the short time region, and concentration profiles are also reported.
\end{abstract}

Recently a great effort has been made to understand the electrochromic process which occurs at $\mathrm{WO}_{3}$ electrodes during reduction and reoxidation and to construct display devices based on this process (1-5). While it appears clear that the process involves formation and oxidation of hydrogen tungsten bronzes, the detailed mechanism and a quantitative model of the electrochromic process and the steps which govern the rate of the color-bleach (CB) process have not been resolved. Different $\mathrm{WO}_{3}$ films produced by vacuum evaporation exhibit different response times for coloring and bleaching, even when they are prepared by similar techniques (5). Moreover different types of $\mathrm{WO}_{3}$ electrodes (e.g., anodic $v s$. evaporated films) show significant differences in response time and electrochemical characteristics in the electrochromic region (6-8). The existence of water in the $\mathrm{WO}_{3}$ film

- Electrochemical Society Active Member

Key words: electrode, interfaces, films.
(7-9) and film porosity (7-8) appear to play important roles in determining the response time of the $\mathrm{WO}_{3}$ electrodes. Crandall and Faughnan (10) discussed the factors entering into the dynamics of the $C B$ process at $\mathrm{WO}_{3}$ and compared values for the composition of the film with time, obtained during potential-step experiments, with calculated values. Their model assumed that mass transfer within the film was very large and that the rate-limiting steps involved proton transfer at the $\mathrm{WO}_{3}$ /liquid interface and the buildup of a "back emf" as the hydrogen bronze formed. Good agreement between the experimental and calculated values was obtained in the short time region after the start of the coloring step or when the coloring step was made at low potentials within the electrochromic region. With this model (5) the bleaching process is limited by the "space charge" which is created by accumulation of $\mathrm{H}^{+}$in the film. Arnoldussen (11) measured exchange currents and transfer coefficients for 